\title{
Bladder dysfunction and neurological disability at presentation in closed spina bifida
}

\author{
L B Johnston, M Borzyskowski
}

\begin{abstract}
Congenital closed spinal anomalies are associated with distortion of the spinal cord, the spinal nerve roots or both, and can result in neurological abnormalities of the lower limbs and neuropathic bladder dysfunction. This study reports clinical and videourodynamic findings in a group of 51 patients with closed spina bifida. The mean age at presentation to a specialist neurourological clinic was 3.3 years. Twenty five patients presented with urinary tract disturbance and 12 presented with neurological problems. Thirty three had normal neurological examination or only minor objective signs, 21 had normal renal tract ultrasonography but only two patients had normal videourodynamics, with 31 having two or more abnormalities during this assessment. Neither clinical neurological assessment nor the history of voiding behaviour are reliable indicators of bladder dysfunction and subsequent risk of renal damage. Therefore, all patients with a known or suspected diagnosis of closed spina bifida should have videourodynamic assessment.

(Arch Dis Child 1998;79:33-38)
\end{abstract}

Keywords: closed spina bifida; videourodynamics; neuropathic bladder dysfunction

Closed spina bifida (a skin covered congenital cord fusion defect) consists of a heterogeneous group of developmental anomalies that include meningocele, lipomeningocele, primary tethered cord, diastematomyelia, intradural lipoma, dermoid cyst, syrinx, and sacral agenesis. ${ }^{1}$ These abnormalities result in distortion of the developing neural tissues, with subsequent damage of the spinal cord and/or nerve roots, and should be differentiated from simple failure of fusion of the vertebral arches of L5 and $\mathrm{S} 1$ in the normal population, which is not associated with spinal cord pathology. ${ }^{1}$ Pathological closed spinal defects may be heralded at birth by a skin lesion, such as a lipoma, hairy tuft, or naevus overlying the spine, or a limb deformity resulting from the neurological abnormality. However, it might not be recognised until the onset of urinary incontinence, urinary tract infections (UTIs), or orthopaedic problems in later childhood when there has been growth of the spine or increased mobility. Often, there is no clinical neurological abnormality or only a minor deficit, which has not been recognised by the parents or the child. The mechanism for deterioration with age is unclear, but it is suggested that growth with the cord pathologically attached within the spinal column might cause ischaemic spinal cord damage. ${ }^{2}$ The resultant neuropathic bladder problems may directly cause injury to the kidneys. $^{3-6}$

Therefore, our study describes the neurourological problems seen at presentation of children with closed spinal defects who were referred to a specialist paediatric neurourology centre. Videourodynamic studies form part of the routine investigation of these patients and our findings highlight the range and severity of the renal and bladder dysfunction at presentation in this predominantly ambulant population.

\section{Methods}

Children with closed spina bifida who were investigated between 1976 and 1995 at the paediatric neurourology clinic at Guy's Hospital, London were the basis of this study. Their notes were reviewed to describe their neurological, orthopaedic, and urological problems when they first presented. All patients underwent routine assessment of the urinary tract, including a full history with particular attention to bladder habit and any history of UTI. Videourodynamic assessment of the bladder and urethra, using standard techniques, and premicturation and postmicturition renal tract ultrasound scans were performed in each case. ${ }^{78}$ Bladder compliance (filling volume in millilitres divided by the end filling detrusor pressure), detrusor hyperreflexia (involuntary contractions during bladder filling in the presence of a known neurological disorder), distal urethral sphincter activity, and the presence of vesicoureteric reflux were assessed during videourodynamic testing. Such studies have been performed on children in this hospital for the past 19 years and the reproducibility of the technique has been well established. ${ }^{9}{ }^{10}$ Dimercaptosuccinic acid scans were performed in cases with a history of UTI to detect renal scars. The level and nature of the spinal lesion was defined by spinal radiography and magnetic resonance imaging (MRI), or spinal myelography in earlier cases.

\section{Results}

REASONS FOR REFERRAL

Fifty one patients (32 girls, 19 boys) with closed spina bifida were identified. The age range at referral was 6 months to 10 years (mean, 3.3 years; median, 3). These patients were referred for a variety of reasons. Most had urinary tract problems (17 were incontinent, six had recurrent UTIs, and two had abnormal renal tract investigations at other hospitals). 
Table 1 Spinal pathology

\begin{tabular}{lc}
\hline Spinal pathology & $\begin{array}{l}\text { Number of } \\
\text { patients }\end{array}$ \\
\hline Meningocele & 7 \\
Lipomeningocele & 1 \\
Sacral agenesis & 5 \\
Dysraphism and intraspinal lipoma & 2 \\
Syrinx & 2 \\
Dysraphism & 1 \\
Tethered cord with no other intraspinal & \\
$\quad$ abnormality & 4 \\
Tethered cord secondary to & 13 \\
$\quad$ Intraspinal lipoma & 2 \\
$\quad$ Syrinx & 4 \\
Diastematomyelia & 4 \\
$\quad$ Lipomeningocele & 6 \\
Others & \\
\hline
\end{tabular}

One patient was referred for investigation of faecal incontinence, five for videourodynamic assessment following spinal surgery, and eight for further investigation after discovery of a dermatological abnormality overlying their lower spine. Twelve were referred as a result of neurological deterioration and one with delay in development of motor milestones. Seven of these patients were referred following spinal surgery without preoperative videourodynamic assessment.

\section{CLINICAL FINDINGS}

Eleven patients had no cutaneous stigmata of closed spina bifida on their backs, but 27 patients had a swelling ( 15 were lipomas), four had a hairy tuft and dimple, three each had either a naevus or a scar, one had a sacral pit. Thus, 38 of 51 patients had a visual sign identifiable at birth. The nature of the pathological abnormalities is described in table 1 and the spinal level involved is shown in fig 1 . Two

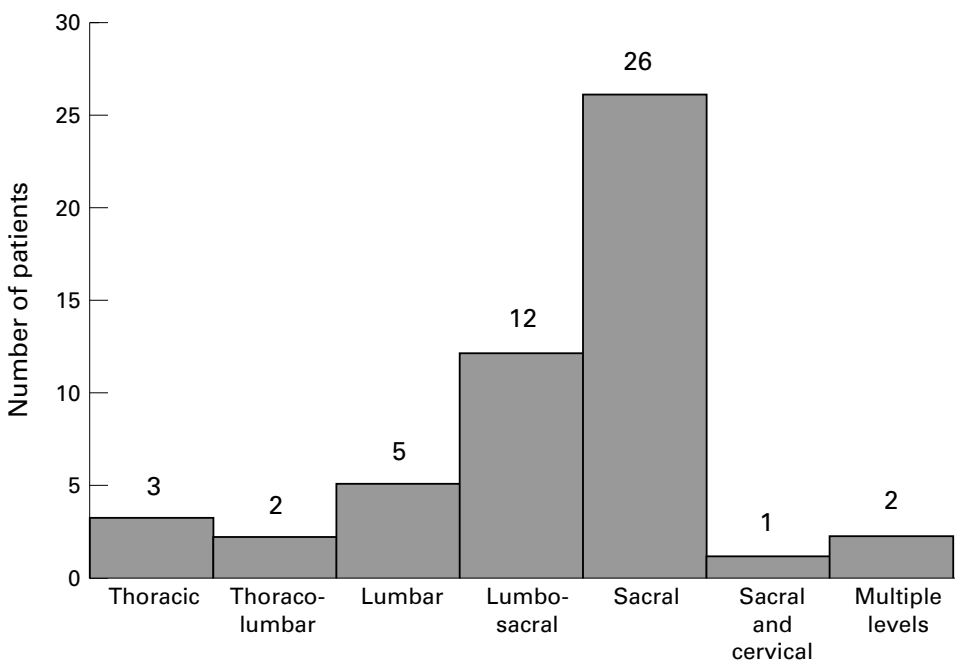

Figure 1 Level of the spinal lesion.

Table 2 Bladder and bowel habit at time of presentation

\begin{tabular}{lcrlll}
\hline & \multicolumn{5}{l}{ Bowel } \\
\cline { 4 - 6 } Bladder & Patients (n) & UTI & Constipation & Incontinance & Normal \\
\hline Normal & 4 & 1 & 0 & 0 & 4 \\
Nappies & 17 & 8 & 3 & 0 & 14 \\
Incontinent & 25 & 15 & 12 & 4 & 9 \\
Urge and frequency & 3 & 2 & 0 & 0 & 3 \\
Retention & 2 & 2 & 1 & 0 & 1 \\
\hline
\end{tabular}

patients had hydrocephalus, of whom one had required ventriculoperitoneal shunting before referral.

At the time of referral, 18 of these patients had normal intellectual development and attended mainstream schools, four attended special needs schools (two of these patients had Down's syndrome), and of the 29 patients who were preschool, none had special educational needs. Thirty seven patients had normal mobility without any orthopaedic aids, 13 required assistance in the form of ankle foot orthoses, and only one was wheelchair bound. At presentation, the neurological examination was entirely normal in eight patients, and 25 had only minor objective motor or sensory signs, such as abnormal reflexes, muscle wasting, or reduced power. Talipes equinovarus was present at birth in six cases; one patient was born with arthrogryposis. Ten patients developed neurological problems in later childhood; four with unilateral lower limb shortening, five with pes cavus, and one with footdrop. Two patients had scoliosis in addition to their other abnormal clinical findings.

At referral, only four patients had normal urinary and faecal continence after toilet training and on detailed questioning one of these patients had had a UTI. Seventeen patients were still in nappies, appropriately for their age, and there was no observed abnormality in their bladder habit. However, three of these patients were constipated and eight had suffered UTIs. At the time of first presentation, 30 patients had abnormal bladder habit, 25 were incontinent, three were dry but had urge and frequency, and two had urinary retention. Of these 30 children with abnormal voiding behaviour, 13 were constipated, four had faecal incontinence, and 19 had suffered at least one UTI (table 2).

\section{INVESTIGATION FINDINGS}

At the time of referral, 21 patients had normal ultrasonography of the renal tract, while 14 showed evidence of dilated upper renal tracts, six showed residual volume postmicturition, six had thick walled bladders, two had a unilateral small kidney, and one patient had renal scars. At presentation, the dimercaptosuccinic acid isotope scans revealed that 12 patients already had renal scars (eight unilateral and four bilateral) while 19 had normal examinations.

\section{VIDEOURODYNAMIC FINDINGS}

Compliance (filling volume in millilitres divided by the end filling detrusor pressure) was decreased in 21 patients in our group. Detrusor sphincter dyssynergia (detrusor contraction concurrent with involuntary contraction of the external urethral sphincter) was seen in 22 patients, and a fixed (non-relaxing) distal sphincter was present in a further five cases. Vesicoureteric reflux was demonstrated in 13 patients. Forty two patients had detrusor hyperreflexia (involuntary contractions during bladder filling in the presence of a known neurological disorder) and 35 patients failed to empty their bladders fully. The results for the above criteria are compared with the reported 
Table 3 Videourodynamic findings compared with bladder habit

\begin{tabular}{|c|c|c|c|c|c|c|c|c|c|c|c|c|}
\hline \multirow[b]{2}{*}{$\begin{array}{l}\text { Bladder } \\
\text { habit }\end{array}$} & \multicolumn{2}{|c|}{ Compliance } & \multicolumn{3}{|c|}{ Urethral sphincter } & \multicolumn{2}{|c|}{ VU reflux } & \multicolumn{2}{|c|}{ Bladder emptying } & \multicolumn{3}{|c|}{ Detrusor hyperreflexia } \\
\hline & Normal & Decreased & Normal & Fixed & $D S D$ & None & Present & Complete & Poor & None & $\begin{array}{l}<50 \mathrm{~cm} \\
\mathrm{H}_{2} \mathrm{O}\end{array}$ & $\begin{array}{l}\geqslant 50 \mathrm{~cm} \\
\mathrm{H}_{2} \mathrm{O}\end{array}$ \\
\hline Wet & 15 & 10 & 7 & 4 & 14 & 16 & 9 & 4 & 21 & 5 & 8 & 12 \\
\hline Urge & 2 & 1 & 2 & 0 & 1 & 3 & 0 & 2 & 1 & 0 & 1 & 2 \\
\hline Retention & 1 & 1 & 1 & 1 & 0 & 2 & 0 & 0 & 2 & 0 & 1 & 1 \\
\hline Nappies & 9 & 8 & 10 & 0 & 7 & 13 & 4 & 6 & 11 & 2 & 4 & 10 \\
\hline Dry & 3 & 1 & 3 & 1 & 0 & 4 & 0 & 4 & 0 & 1 & 2 & 1 \\
\hline Total & 30 & 21 & 23 & 6 & 22 & 38 & 13 & 16 & 35 & 8 & 16 & 26 \\
\hline
\end{tabular}

VU, vesicoureteric; DSD, detrusor sphincter dyssynergia.

bladder habit in table 3. Only two patients had completely normal videourodynamic studies, one was 2 years old and continent, the other was only 6 months of age and still in nappies, with no observed abnormality in bladder voiding.

\section{MANAGEMENT}

Management was tailored for the individual, using the results of the videourodynamic tests. Thirty of the children in this group have successfully used clean intermittent catheterisation to improve their continence and only a small number (six patients) have remained dry without medical treatment. Anticholinergic agents and prophylactic antibiotics were used as indicated. In this group of patients, nine have required urological surgery (four needed ureteric reimplantation and five needed caecocystoplasty). Twenty two have had spinal cord surgery because of neurological or neurourological deterioration.

\section{Discussion}

Normal bladder function relies on complex neurophysiological interactions between sacral spinal nerves and the cerebral cortex. Congenital spinal abnormalities interfere with this system and can result in bladder dysfunction in addition to lower limb neurological abnormalities. Closed spina bifida refers to skin covered congenital cord fusion defects and includes a number of discrete pathological entities. These result in similar clinical syndromes with different degrees of bladder dysfunction and neurological deficit. Any congenital intraspinal abnormality can result in tethering, a pathological fixation of the spinal cord within the spinal canal, which interferes with the normal postnatal ascent of the conus medullaris, most significantly in the first few months of life. This can result in ischaemic damage to the spinal cord with subsequent neurourological and neurological dysfunction. The clinical picture depends on the level of the lesion more than its exact nature, with sacral lesions being compatible with excellent mobility but severe bladder dysfunction. ${ }^{411} 12$

Our patients had predominantly low spinal lesions with 43 being lumbar and/or sacral. Only nine children in this group were diagnosed in the first year of life and only one was diagnosed immediately after birth. As a result of normal or near normal mobility and the difficulty in recognising bladder dysfunction before toilet training, referral was not made until after 3 years of age in half of these cases, and in some the diagnosis of spina bifida had not yet been made. Urinary incontinence and UTIs were the most frequent reason for referral but in about one quarter of the children a deterioration in neurological status brought the patient to medical attention.

All patients were ambulant except one and only a quarter required orthopaedic aids in the form of ankle foot orthoses. Only four children had special educational needs and of these two had Down's syndrome. Detailed neurological examination was entirely normal in eight patients and revealed only minor abnormalities in a further 25 patients, which were not associated with any subjective deficit. This is consistent with most of the defects being low lying because sacral lesions produce few neurological findings in the lower extremities. Bladder function, which is controlled intricately by sacral nerves, may be the first sign of these low spinal cord abnormalities. ${ }^{13}$ Indeed, more than half of our patients had abnormal voiding behaviour at the time of presentation but a further third of the patients had not started toilet training and only two patients had completely normal videourodynamic studies (one 2 year old who was trained and a 6 month old). The videourodynamics in the rest of the group demonstrated that these children with closed spinal defects suffer the full spectrum of lower urinary tract dysfunction seen in children with open spina bifida, despite their good mobility. This demonstrates that neither the history of voiding habits nor the neurological examination are reliable indicators of bladder dysfunction, which is consistent with the findings of Mevorach et al and Dator et al in children with myelomeningocele. ${ }^{411}$ In particular, one cannot be reassured by a normal neurological clinical examination; in our series only one child of the eight with normal neurology had a normal videourodynamic study, although three others reported normal voiding patterns.

The predictive value of videourodynamic studies is now well established. ${ }^{14-17}$ The factors associated with upper tract deterioration are vesicoureteric reflux, high intravesical pressures (because of poor bladder wall compliance or detrusor muscle hyperreflexia), and detrusor sphincter dyssynergia, particularly if they occur together or in the presence of infected urine. $^{9141518}$ The presence of residual urine after incomplete bladder emptying increases the risk of UTI. An American group have used these features, which are all measured during videourodynamic studies, to design an objec- 
tive scoring system, the hostility score, to quantify the risk to the kidneys of lower urinary tract dysfunction. ${ }^{15}$ In addition, Steinhardt et al found consistent and significant decreases in glomerular filtration rate at high bladder pressures in five myelodysplasic patients. ${ }^{19}$ Therefore, videourodynamic studies provide a useful objective measurement of bladder and urethral function, which can assess the potential risk of renal damage and allow comparison with future studies of the same patient to monitor the effect of treatment. They are a sensitive indicator of change and can detect deterioration early, often before any change in clinical neurological or bladder status.

The patients in our study had significant abnormalities found on videourodynamic testing at presentation: 13 had vesicoureteric reflux, 42 had detrusor hyperreflexia, and 22 had detrusor sphincter dyssynergia with a further six having non-relaxing distal sphincters. Thirty one patients had a combination of at least two of these abnormalities, making their bladders dangerous in terms of the potential risk of renal damage. This is reflected in the high incidence of renal scarring at presentation. All of these children with renal scars had suffered at least one UTI, underlining the importance of preventing UTI in children with dangerous neuropathic bladders to prevent the morbidity associated with renal scarring. Upper urinary tract involvement and renal impairment may already be present at the time the first bladder problem is manifest, which makes early diagnosis of the spinal defect and subsequent videourodynamic assessment extremely important. ${ }^{3}$

There has been some debate over the timing of initial videourodynamic studies and the predictive value of performing these in infancy. Bauer et al reported a study of 36 infants, 18 with detrusor sphincter dyssynergia, of whom 13 later developed hydronephrosis. This occurred in only one of the nine with no sphincter activity. ${ }^{20}$ The hydronephroses of these affected infants were managed successfully by vesicostomy or clean intermittent catheterisation which ensured regular bladder emptying. Other reports confirm the finding that raised intravesical pressure and detrusor sphincter dyssynergia detected in infancy is associated, as in older children, with a significant risk of developing upper renal tract changes. ${ }^{17}{ }^{21}$ When closed spina bifida is diagnosed, videourodynamics should be performed so that bladder problems and their potential for renal damage can be identified as early as possible. ${ }^{8}$ Renal ultrasound scanning and micturating cystography are helpful investigations but cannot exclude severe bladder pathology and do not measure intravesical pressure, so are no substitute for videourodynamic studies. In this study, where most of patients had at least one abnormality on videourodynamic testing, less than half were reported to have a normal renal ultrasound scan.

Thus, optimum management requires early diagnosis of the spinal abnormality, which may be aided in the neonatal period by an overlying cutaneous lesion or a lower limb orthopaedic

\section{Key messages}

- Clinical signs are often missed in newborns, delaying diagnosis until later childhood when patients present with urinary incontinence, failure of toilet training, urinary tract infection, or orthopaedic problems.

- Neither the clinical neurological deficit, which tends to be minor, nor the history of voiding habit are reliable indicators of the severity of bladder dysfunction and subsequent risk of renal damage.

- Videourodynamics demonstrated that children with closed spina bifida suffer the full spectrum of lower urinary tract dysfunction seen in children with open spina bifida, despite their good mobility: in this study fewer than half of patients had a normal renal tract ultrasound scan but most had at least one abnormality on videourodynamic testing.

- Upper urinary tract involvement and renal impairment may already be present when the first bladder problem is manifest: a quarter of cases in our study had renal scarring at presentation.

abnormality. Our study confirms that the diagnosis is often missed in newborns who do not present until urinary incontinence, failure of toilet training, UTI or orthopaedic problems supervene in later childhood. Once the diagnosis is suspected, these patients should receive the same urgent attention as children diagnosed in the neonatal period. Talipes equinovarus was present in six of our patients, but led to neonatal diagnosis of spina bifida in only one child, the other five children undergoing treatment of their talipes without the underlying diagnosis being made. Spinal ultrasound in the neonatal period can be helpful in diagnosis, but MRI scanning is recommended to detail the spinal pathology. ${ }^{22}$ MRI serves not only to diagnose but also provides a baseline for future comparison in cases where tethering, or pathological fixation within the spinal canal of the spinal cord is suspected. ${ }^{23}$ Spinal cord imaging should be accompanied by urine culture, premicturation and postmicturition ultrasound imaging of the renal tract, and referral for neurourological assessment, including videourodynamic testing.

The natural history of the neurourology of closed spina bifida necessitates continued surveillance of all children affected, as relatively safe bladders can deteriorate and become a danger to the kidneys. Lais et al followed 148 children with myelomeningocele and $40 \%$ showed evidence of deterioration during the first two years of life when growth is at its most rapid. ${ }^{24}$ Some studies of "occult" spinal defects are misleading, in that they suggest a significant chance of stability and even improvement in some cases. ${ }^{62526}$ These studies include many individuals with simple failure of fusion of the vertebral arches of L5 and S1, which has been 
found on plain radiograph in children with enuresis. However, this is a normal variant found in up to $10 \%$ of the normal population. ${ }^{15}$ Most authors recommend serial screening with videourodynamics, but some studies have also used electrophysiological testing to demonstrate the natural progression of neurourological dysfunction in closed spina bifida. ${ }^{13}$ Keating and colleagues $^{27}$ and Satar and colleagues ${ }^{28}$ report series of closed spinal defects presenting at various ages. Both authors conclude that the neurourology is variable, progressive (in that older patients present with more severe bladder dysfunction), but might be reversible, although this reversibility diminishes with age. These facts underlie the recommendation of early referral to a specialist paediatric neurourology centre for thorough investigation. Clinical, radiological, and videourodynamic assessments are then performed to define the neurourological pathophysiology and provide a guide for management and a baseline for future comparison. ${ }^{13}$

Although incontinence is a major social handicap, the danger to the kidneys of neuropathic vesicourethral dysfunction is of crucial importance. Therefore, the aims of management are: (1) to preserve normal renal function and prevent damage to the kidneys, (2) to achieve urinary and faecal continence, and (3) to allow the patient to lead an independent life and develop a sense of self esteem. ${ }^{18}$ Videourodynamics enable management to be tailored to each individual's needs. Clean intermittent catheterisation, to ensure regular bladder emptying, is the mainstay of the treatment of patients with poorly emptying, high pressure bladders, and is combined with medical treatment as appropriate. Gerianotis et al have shown that this reduces the risk of upper urinary tract dysfunction in treated infants and children compared with those managed conservatively. ${ }^{29}$ Neuropathological bowel disturbance is common in these patients and might provide a diagnostic clue; furthermore, adequate treatment improves the efficacy of medical management of the bladder dysfunction. ${ }^{1}$ The management of bowel disturbance can be challenging and it might be useful to involve a paediatric gastroenterologist. Prompt neurosurgery might prevent progression of the symptoms but, with a risk of neurourological deterioration, videourodynamic assessment should always be carried out postoperatively. $^{230}$ The results of surgery are variable, but some surgical series reported suggest a chance of recovery, and this effect is more pronounced in younger patients and those with less delay in diagnosis from the onset of symptoms. ${ }^{56162631}$ These series are small and the patients are highly selected, so the evidence is not yet conclusive, but it seems that the potential benefit for postsurgical improvement decreases with advancing age. ${ }^{27} 2832$ The debate over spinal surgery and its timing remains and the problem of late diagnosis and referral are still stumbling blocks in the management of these children.

\section{Conclusions}

The incidence and severity of bladder dysfunction in ambulant children with closed spina bifida is similar to that reported elsewhere in ambulant and non-ambulant children with open spina bifida. Patients with occult spinal defects present with highly dangerous bladders that place the kidneys at risk of irreversible damage. A quarter of cases in this study had renal scarring at presentation. Neither the clinical neurological deficit, which tends to be minor, nor the history of voiding habit are good indicators of the severity of bladder dysfunction. Clinical signs are often missed in the neonatal period, leading to late diagnosis. Early referral to a specialist paediatric neurourology centre is of vital importance for optimal management. Patients require thorough initial neurourological assessment including videourodynamics, preferably in infancy, and careful longitudinal follow up to monitor the natural pathophysiological progression throughout the period of growth. Videourodynamic studies should be performed as soon as the diagnosis of spina bifida is suspected, to define the bladder pathology and direct appropriate treatment. Management should aim to protect the kidneys from the abnormal bladder behaviour. Careful clinical management can minimise deterioration in renal function and enable these patients to lead a full life.

1 Sutherland RS, Mevorach RA, Baskin LS, Kogan BA. Spinal dysraphism in children: an overview and an approach to prevent complications. Urology 1995;46:294-304.

2 Kaplan WE, McLone DG, Richards I. The urological manifestations of the tethered spinal cord. F Urol 1988;140; 1285-8.

3 Borzyskowski M, Neville BGR. Neuropathic bladder and spinal dysraphism. Arch Dis Child 1981;56:176-80.

4 Dator DP, Hatchett L, Dyro FM, Shefner JM, Bauer SB. Urodynamic dysfunction in walking myelodysplastic children. F Urol 1992;148:362-5.

5 Bauer SB, Atala A, Dyro FM, Shefner JM, Shillito J, Scott $\mathrm{RM}$. Urologic manifestations in patients with diastematomyelia [abstract]. F Urol 1992;148:152.

6 Perez LM, Barnes N, McDiarmid SA, Oakes WJ, Webster GD. Urological dysfunction in patients with diastematomyelia. F Urol 1993;149:1503-5.

7 Andersen JT, ed. Urodynamics. Terminology and normal values in children, females and males. Scand f Urol Nephrol 1988;114(suppl):5-19

8 Borzyskowski $M$. An update on the investigation of the child with a neuropathic bladder. Dev Med Child Neurol 1996;38: $744-8$.

9 Mundy AR, Borzyskowski M, Saxon HM. Videourodynamic evaluation of neuropathic vesicourethral dysfunction in children. Br $\mathcal{F}$ Urol 1982;54:645-9.

10 Reid CJD, Borzyskowski M. Lower urinary tract dysfunction in cerebral palsy. Arch Dis Child 1993;68:739-42.

11 Mevorach RA, Bogaert GA, Baskin LS, Lazzaretti CC, Edwards MSB, Kogan BA. Lower urinary tract function in ambulatory children with spina bifida. Br f Urol 1996;77: 593-6.

12 Flanigan RC, Russell DP, Walsh JW. Urologic aspects of tethered cord. Urology 1989;33:80-2.

13 Kothari MJ, Bauer SB, Logigian EL, Shefner JM. Electrophysiological and urodynamic studies to monitor surgical outcome in children with tethered spinal cords. Muscle Nerve 1995;18:1373-7.

14 McLorie GA, Perez-Marero R, Csima A, Churchill BM. Determinants of hydronephrosis and renal injury in patients with myelomeningocele. F Urol 1988;140:1289-92.

15 Galloway M, Mekras JA, Helms M, Webster GD. An objective score to predict upper tract deterioration in myelodysplasia. Urology 1991;145:535-7.

16 Gross AJ, Michael T, Godeman F, Weigel K, Huland $\mathrm{H}$. Urological findings in patients with neurosurgically treated tethered spinal cord. $\mathcal{F}$ Urol 1993;149:1510-11.

17 Perez LM, Khoury J, Webster GD. The value of urodynamic studies in infants less than 1 year old with congenital spinal dysraphism. F Urol 1992;148:584-7.

18 Gamal M, Ghoniem MB, Roach MB, Lewis VH, Harmon EP. The leak pressure and bladder compliance in the urodynamic evaluation of meningocele patients. F Urol 1990;144:1440-2. 
19 Steinhardt GF, Goodgold HM, Samuels LD. The effect of intravesical pressure on glomerular filtration rate in patients

20 Bauer SB, Hallett M, Khoshbin S, et al. Predictive value of urodynamic evaluation in new-borns with myelodysplasia. f Am Med Assoc 1984;252:650-2.

21 Sidi AA, Dykstra DD, Gonzalez R. The value of urodynamic testing in the management of neonates with myelodysplasia: a prospective study. $\mathcal{F}$ Urol 1986;135:90-3.

22 McEnery G, Borzyskowski M, Cox TCS, Neville BGR. The spinal cord in neurologically stable spina bifida: a clinical
and MRI study. Dev Med Child Neurol 1992;34:342-7.

23 Johnson DL, Levy LM. Predicting outcome in the tethered cord syndrome: a study of cord motion. Paediatr Neurosurg 1995;22:115-19.

24 Lais A, Kasabian NG, Dyro FM, Scott RM, Kelly MD, Bauer SB. The neurosurgical implications of continuous neurourological surveillance of children with myelodysplasia. F Urol 1993;150:1879-83.

25 Ritchey ML, Sinha A, Dipietro MA, Huang C, Flood H, Bloom DA. Significance of spina bifida occulta in children with diurnal enuresis. F Urol 1994;152:815-18.
26 Khoury AE, Hendrick EB, McLorie GA, Kulkarni A, Churchill BM. Occult spinal dysraphism: clinical and uro-
dynamic outcome after division of the filum terminale. $\mathcal{f}$ Urol 1990;144:426-9.

27 Keating MA, Rink RC, Bauer SB, et al. Neurourological implications of the changing approach in management of occult spinal lesions. F Urol 1988;140;1299-301.

28 Satar N, Bauer SB, Shefner J, Kelly MD, Darbey MM. The effects of delayed diagnosis and treatment in patients with an occult spinal dysraphism. F Urol 1995;154:754-8.

29 Gerianotis E, Koff SA, Enrile B. The prophylactic use of clean intermittent catheterisation in the treatment of infants and young children with myelomeningocele

30 Zoller G, Schoner W, Ringert RH. Pre- and postoperative urodynamic findings in children with tethered spinal cord syndrome. Eur Urol 1991;19:139-41.

31 Fone PD, Vapnek JM, Litwiller SE, et al. Urodynamic findings in the tethered spinal cord syndrome: does surgical ings in the tethered spinal cord syndrome: does surgical
release improve bladder function? F Urol 1997;157:604-9.

32 Atala A, Bauer SB, Dyro FM, et al. Bladder functional changes resulting from lipomyelomeningocele repair. $\mathcal{F}$ Urol 1992;148:592-4. 